\title{
Particle Motion in Surface Gravity Waves
}

\section{Kern E. Kenyon}

North Lane, Del Mar, USA

Correspondence to: Kern E. Kenyon, kernken@aol.com

Keywords: Surface Gravity Waves, Orbital Particle Motion

Received: January 2, $2021 \quad$ Accepted: February 19, $2021 \quad$ Published: February 22, 2021

Copyright $\odot 2021$ by author(s) and Scientific Research Publishing Inc.

This work is licensed under the Creative Commons Attribution International License (CC BY 4.0).

http://creativecommons.org/licenses/by/4.0/

\section{Open Access}

\section{ABSTRACT}

Fluid particles in translating surface gravity waves have an orbital motion which decreases in size with increasing mean depth. These wave characteristics came from observations and were not forecast theoretically. The classical potential flow model is incapable of explaining the particle movement due to the irrotational assumption and to a flaw in carrying out the method. When a wave passes by an observer from left to right, the particles move clockwise under a crest and a trough. This correct conclusion is consistent with what the incorrect standard theory implies but should not be considered to have been derived from it.

\section{INTRODUCTION}

When a propagating surface gravity wave passes by an observer from left to right, what is to be noticed about the motion of a fluid particle? Small neutrally buoyant particles throughout the water column will help the observer to decide. On my shelf are nine books that discuss the surface gravity wave and include a schematic diagram of the fluid particle's movement. Eight of those diagrams show clockwise rotation under the wave crest (e.g. [1-3]) and one diagram shows counter-clockwise rotation [4]! How is that possible coming from an author better known than most of the other eight?

A short answer is that the draftsman made a mistake that the one author did not catch. However, an alternative possibility for an answer is based on the fact that there is no existing mathematical theory that predicts the nearly circular orbital paths of the fluid particles in translating surface gravity waves nor is the sense of rotation computed. Among the several types of waves in physics, the water wave is the only one that has orbiting material particles, as evidenced by observations.

Rayleigh [5], and a few others, found the steady state reference frame to be enlightening when applied to the translating surface gravity wave. In this frame, the wave shape is stationary and the steady flow passes underneath the wavy surface. But the orbital structure of the fluid particles, found in the more usual fixed reference frame, cannot be recovered from that model and would never have been forecast from it initially. Observations first provided this wave characteristic, and the appropriate physics and mathematics have still not completely caught up to it.

Given the existence of orbital particle motion, a theoretical inference was put forward not too many 
years ago [6] that is outside the classical mathematical framework of the surface gravity wave. Each fluid particle within the depth of wave influence should experience an outward centrifugal force directed from the orbit's center. Since turbulence and wave breaking rarely occur, the centrifugal force must be balanced by an equal but opposite inward force, and in the present circumstance that balancing force can only be a pressure gradient.

This balance of forces led further to the prediction [7] that in a propagating surface gravity wave the orbital velocity and the pressure should decrease at significantly different rates with the increase in depth, whereas the potential flow theory gives the same rate of decay for both variables.

\section{POTENTIAL FLOW}

Beginning in the mid-1800s all scientific explanations of surface gravity waves were founded on one assumption: irrotational motion. As it turns out the original application of this method to water waves has carried along to the present time a fundamental flaw in the reasoning [8] that disqualifies it from any ability to be relevant to understanding the wave's orbital movement of the particles.

First, an ad hoc algebraic compound is dreamed up which will satisfy Laplace's equation: the velocity potential $\varphi$. One factor in the compound is only a function of depth $z$, or $k z$, where $k$ is the wave number; the other factor is a function of a horizontal coordinate $x$ and the time $t$, e.g. $k x-\omega t$, where $\omega$ is the frequency. If the first function is exponential and the second one is sinusoidal, that will work to solve Laplace's equation since the depth decay rate and the spatial propagation rate are both assumed to be proportional to $k$.

Now the velocity potential $\varphi$ is defined such that the horizontal fluid velocity component $u$, for example, is given by $u=\delta \varphi / \delta x$. Setting up a velocity potential that will solve Laplace's equation and is very similar to that presented in many textbooks gives

$$
\varphi=A e^{k z} \sin (k x-\omega t)
$$

where $z$ is measured positive upward from the mean air/water surface and $A$ is a constant. Computing the horizontal velocity component from (1) produces

$$
u=A k e^{k z} \cos (k x-\omega t)
$$

What is the meaning of Equation (2)? It means all the water within the depth of wave influence moves from left to right in the fixed frame (fixed to the bottom or shore of a lake or ocean) at the wave phase speed $c=\omega / k$. That is complete nonsense!

\section{DISCUSSION}

In the fixed reference frame with the surface gravity wave moving from left to right past an observer, which way do the fluid particles rotate under the crest: clockwise or counter-clockwise? Clockwise is the correct answer, and it comes from an incorrect theory.

Two facts are known from observations: the fluid particles have an orbital motion, and the size of the orbits decreases as the mean depth increases. Combining these two facts leads to the idea that at the top of an orbit under a crest the forward speed is a small bit faster than the return speed is at the bottom of the orbit under a trough. Consequently after each wave period there is a small net displacement forward of each fluid particle. Forward points in the direction of wave propagation. This is commonly called the Stokes drift and its significance is: these waves have linear momentum in addition to the self-evident orbital angular momentum of each fluid particle. It would not be reasonable to think that the linear momentum points in the direction opposite to that of wave travel. Stokes made the correct prediction from an incorrect theory!

Energies of the surface gravity waves, as classically calculated, have been disrupted, partially by the irrotational assumption, in the sense that entirely different methods were used for deriving the potential and kinetic energies [9]. It might turn out that these old energy formulas will not agree well when compar- 
isons with data occur in the future.

\section{CONCLUSION}

It is concluded that there is no existing theory, classical or more recent, which predicts that the motion of the fluid particles in a propagating surface gravity wave will be orbital, that the size of the orbits decreases with increasing depth, or that the sense of orbital motion is clockwise under a crest and a trough when the wave passes an observer from left to right. These wave characteristics have all come from observations. Given the orbital motion, certain theoretical inferences have been made, such as the outward centrifugal force on each orbiting particle which must be balanced by an equal inward pressure gradient, and that the diminishing orbital radius down in the water column implies that these waves have linear momentum as well as orbital angular momentum.

\section{ACKNOWLEDGEMENTS}

Tony Sturges made a pertinent comment about the Stokes drift.

\section{CONFLICTS OF INTEREST}

The author declares no conflicts of interest regarding the publication of this paper.

\section{REFERENCES}

1. Lamb, H. (1932) Hydrodynamics. 6th Edition, Dover Publications, New York.

2. Lighthill, J. (1978) Waves in Fluids. Cambridge University Press, Cambridge.

3. Faber, T.E. (1995) Fluid Dynamics for Physicists. Cambridge University Press, Cambridge. https://doi.org/10.1017/CBO9780511806735

4. Defant, A. (1961) Physical Oceanography. Pergamon Press, New York, Vol. II.

5. Rayleigh, L. (1876) On Waves. The London, Edinburgh, and Dublin Philosophical Magazine and Journal of Science, 1, 257-279.

6. Kenyon, K.E. (1991) Cyclostrophic Balance in Surface Gravity Waves. Journal of the Oceanographical Society of Japan, 47, 45-48. https://doi.org/10.1007/BF02301754

7. Kenyon, K.E. (2013) Depth Decay Rate for Surface Gravity Wave Pressure and Velocity. Natural Science, 5, 44-46. https://doi.org/10.4236/ns.2013.51007

8. Kenyon, K.E. (2020) Frictionles Surface Gravity Waves. Natural Science, 12, 199-201. https://doi.org/10.4236/ns.2020.124017

9. Kenyon, K.E. (2020) On Surface Gravity Wave Energies. Natural Science, 12, 1-3. https://doi.org/10.4236/ns.2020.121001 